\title{
Prospective study of changes in sugar-sweetened beverage consumption and the incidence of the metabolic syndrome and its components: the SUN cohort
}

\author{
María T. Barrio-Lopez ${ }^{1,2}$, Miguel A. Martinez-Gonzalez ${ }^{1,3 *}$, Alejandro Fernandez-Montero ${ }^{1}$, \\ Juan J. Beunza ${ }^{1}$, Itziar Zazpe ${ }^{1,4}$ and Maira Bes-Rastrollo ${ }^{1}$ \\ ${ }^{1}$ Department of Preventive Medicine and Public Health, School of Medicine, University of Navarra, Spain Universidad de \\ Navarra Ed. Investigación, C/Irunlarrea, 1, 31008 Pamplona, Navarra, Spain \\ ${ }^{2}$ Department of Cardiology and Cardiac Surgery, University Clinic of Navarra, Avenida de Pio XII, 36, 31008 Pamplona, \\ Navarra, Spain \\ ${ }^{3}$ CIBERobn, Instituto de Salud Carlos III, Pamplona, Navarra, Spain \\ ${ }^{4}$ Department of Nutrition and Food Sciences, Physiology and Toxicology, School of Pharmacy, University of Navarra, \\ Irunlarrea, 1, 31008 Pamplona, Navarra, Spain
}

(Submitted 22 August 2012 - Final revision received 14 February 2013 - Accepted 14 February 2013 - First published online 27 March 2013)

\begin{abstract}
The incidence of the metabolic syndrome (MetS) is increasing and lifestyle behaviours may play a role. The aim of the present study was to prospectively assess the association between changes in the consumption of sugar-sweetened beverages (SSB) and the incidence of the MetS and its components in a Spanish cohort of university graduates. We included 8157 participants initially free of the MetS and followed up during at least 6 years. SSB consumption was collected by a FFQ previously validated in Spain. The change in SSB consumption was calculated as the difference between SSB consumption at a 6-year follow-up and baseline consumption. The MetS was defined according to the International Diabetes Federation and the American Heart Association/National Heart, Lung, and Blood Institute's new definition of the MetS that had harmonised previous definitions. The associations between changes in SSB intake and the MetS were examined using multiple logistic regression. We observed 361 incident cases of the MetS. Participants who increased their consumption of SSB (upper $v$. lower quintile) had a significantly higher risk of developing the MetS (adjusted OR 2.2, $95 \%$ CI 1.4, 3.5; $P$ for trend=0.003). Similarly, they presented a significantly higher risk of developing high blood pressure (adjusted OR 1.6, $95 \%$ CI 1.3, 2.1), central obesity (adjusted OR 2.3, 95\% CI 1.9, 2.7), hypertriacylglycerolaemia (adjusted OR 1·7, $95 \%$ CI $1 \cdot 1,2 \cdot 6$ ) or impaired fasting glucose (adjusted OR 1.6, 95\% CI 1·1, $2 \cdot 2)$. In conclusion, an increase in SSB consumption was associated with a higher risk of developing the MetS and other metabolic disorders after 6 years of follow-up in a Mediterranean cohort of university graduates.
\end{abstract}

Key words: Sugar-sweetened beverages: Metabolic syndrome: Cohorts: Prospective studies

The metabolic syndrome (MetS) is considered to be a clustering of metabolic alterations conferring a high risk of developing type 2 diabetes and CVD, and a higher risk of all-cause mortality. The prevalence of the MetS differs according to the criteria used to define it. In developed countries, the prevalence of the MetS reaches about $25 \%$ of the population ${ }^{(1-7)}$, and its incidence has been increasing over the last years.

However, there is a heterogeneous distribution of the MetS across different countries and races studied ${ }^{(5-7)}$. In Spain, the prevalence of the MetS has apparently increased in the past years from $10 \cdot 2 \%$ in $2005^{(8)}$ to $31 \%$ in $2012^{(9)}$. These differences in prevalence cannot be explained by population genetics alone. Environmental influences may play an important role.
Low physical activity, smoking, low social class and low educational level have been associated with an increased risk of the $\operatorname{MetS}^{(8,10)}$. Food habits may also be one of the most important factors determining the MetS. Although solid food is an important source of nutrients in the diet, drinks can be a major source of carbohydrates as well. Some of them contain sugar, sweeteners, alcohol or caffeine ${ }^{(11)}$ with the potential to increase the risk of the MetS.

Several reports have indicated an increasing consumption of soft drinks among children, adolescents and adults over the past three decades ${ }^{(12,13)}$. Even though, subsequently, in recent years, a mild decrease in sugar-sweetened beverages (SSB) has been described ${ }^{(14)}$. Many clinical studies have

Abbreviations: MetS, metabolic syndrome; SSB, sugar-sweetened beverages; SUN, Seguimiento Universidad de Navarra. 
linked the rising consumption of soft drinks to an increase in obesity and diabetes mellitus among children and adolescents $^{(15-18)}$, and to the development of hypertension ${ }^{(19)}$ and $\mathrm{CVD}^{(20)}$ in adults. There is no consensus in the literature on the association between soft drink consumption and TAG levels ${ }^{(21-25)}$; however, some studies have found a negative association between soft drink consumption and serum HDL-cholesterol ${ }^{(20,26,27)}$

The Oslo Health Study found that soft drink consumption was positively associated with the prevalence of the MetS, and this cross-sectional association was independent of the presence or absence of sugar in soft drinks and a large number of possible confounders ${ }^{(28)}$. Prospective studies about this topic are scarce. There are only three prospective studies that have shown a link between soft drink consumption and the risk of developing the complete $\operatorname{MetS}^{(26,29,30)}$. All of them were summarised in a meta-analysis ${ }^{(31)}$. However, none of these studies has been conducted in the context of a Mediterranean dietary pattern where the average consumption of SSB is very low in comparison with the USA or North European countries. Additionally, all previous studies only assessed baseline consumption, but not the changes in the consumption of soft drinks during follow-up.

The aim of the present study was to assess the association between changes in SSB consumption and the incidence of the MetS and its components in a cohort of Spanish university graduates.

\section{Experimental methods}

\section{Study sample}

The Seguimiento Universidad de Navarra (SUN) Project is a dynamic prospective cohort study conducted in Spain with permanently open recruitment and whose participants are all university graduates who are contacted and followed up using mailed or Web-based questionnaires. A detailed description of study methods has been published previously ${ }^{(32,33)}$. Briefly, beginning in December 1999, all graduates of the University of Navarra, registered nurses from some Spanish provinces and university graduates from other colleges and associations received a mailed questionnaire and a letter of invitation to participate in the SUN Project.

The present study was conducted according to the guidelines laid down in the Declaration of Helsinki, and all procedures involving participants were approved by the Institutional Review Board of the University of Navarra. A response to the initial questionnaire was assumed to be a surrogate of detailed informed consent that it implied the willingness to participate in the study. For this purpose, this informed consent was obtained from all participants.

After baseline assessment, participants received follow-up questionnaires every 2 years that contained a wide variety of questions on diet, lifestyle, risk factors and medical conditions.

For the present MetS study, only a subsample of the cohort was selected. To warrant a minimum follow-up of 6 years, we included only those participants who had completed questionnaires up to 6 years of follow-up. A total of 14716 SUN participants responded to the baseline questionnaire before September 2004. Among them, 3728 participants were excluded because they had one or more criteria for the MetS. Another 1139 participants with extreme energy intake $(<3349 \mathrm{~kJ} / \mathrm{d}$ $(<800 \mathrm{kcal} / \mathrm{d})$ for men or $<2093 \mathrm{~kJ} / \mathrm{d}(<500 \mathrm{kcal} / \mathrm{d})$ for women, $>16747 \mathrm{~kJ} / \mathrm{d}(>4000 \mathrm{kcal} / \mathrm{d})$ for men or $>14654 \mathrm{~kJ} / \mathrm{d}$ $(>3500 \mathrm{kcal} / \mathrm{d})$ for women) were also excluded. Among the remaining, 1136 participants answered some follow-up questionnaire but not the 6-year or 8-year follow-up questionnaire, and 556 did not answer any follow-up questionnaires. Therefore, 8157 participants were included in the final analyses.

\section{Definition and components of the metabolic syndrome}

We defined the MetS according to the International Diabetes Federation and American Heart Association/National Heart, Lung, and Blood Institute's new definition of the MetS that has harmonised all previous definitions ${ }^{(34)}$. According to this definition, the diagnosis of the MetS needs the presence of three of any of five risk factor components. These five components are as follows: (1) elevated waist circumference according to the population and country-specific definition (in the present study: $\geq 94 \mathrm{~cm}$ in males and $\geq 80 \mathrm{~cm}$ in females); (2) elevated TAG ( $\geq 1500 \mathrm{mg} / \mathrm{l})$ or presence of drug treatment for elevated TAG; (3) reduced HDL-cholesterol $(<400 \mathrm{mg} / 1$ in males and $<500 \mathrm{mg} / 1$ in females) or presence of drug treatment for reduced HDL-cholesterol; (4) elevated blood pressure (systolic $\geq 130$ and/or diastolic $\geq 85 \mathrm{mmHg}$ ) or presence of antihypertensive drug treatment in a patient with a history of hypertension; (5) elevated fasting glucose $(\geq 1000 \mathrm{mg} / \mathrm{l}$ ) or drug treatment for elevated glucose.

In the 6- and 8-year follow-up questionnaires, self-reported data about these specific MetS criteria were collected. A measuring tape was sent via ordinary mail to all participants with the 6- or 8-year follow-up questionnaire including an explanation about how to measure their own waist. The validation of the self-reported data of the criteria needed to classify participants into the MetS was assessed in a subsample of the cohort, finding significant intraclass correlation coefficients between 0.5 and $0.9(P<0.001)$ depending on the criteria between each self-reported MetS component and their direct assessments by an experienced physician ${ }^{(35)}$. In another validation study also conducted in a subsample of this cohort on the complete MetS itself, we found a proportion of confirmed MetS of $91 \cdot 2 \%$ (95\% CI 80.7, 97.1) and non-confirmed MetS of $92 \cdot 2 \%(95 \%$ CI $85 \cdot 1,96 \cdot 4)$ between the self-reported diagnosis of the MetS and the MetS diagnosed by the medical records of our participants ${ }^{(36)}$.

Incident cases of the MetS were defined as those participants who did not have the MetS at baseline, and they reported criteria of the MetS in either the 6- or 8-year follow-up questionnaire.

\section{Assessment of dietary and non-dietary exposures}

Dietary habits at baseline were assessed using a semi-quantitative FFQ with 136 items, previously validated in Spain ${ }^{(37)}$ and recently re-evaluated ${ }^{(38,39)}$. We defined an 'a priori' 
Mediterranean dietary pattern using the 0 (minimum) to 9 (maximum) score proposed by Trichopoulou et al. ${ }^{(40)}$. The baseline questionnaire also included different questions related to lifestyle. Sociodemographic variables (sex, age, years of university education and employment), anthropometric data, health-related habits (e.g. smoking status, alcohol consumption and physical activity) and medical history information (medication use, cholesterol level, blood pressure and family history of several diseases) were also collected. The reproducibility and validity of self-reported anthropometrics $^{(41)}$, the physical activity questionnaire ${ }^{(42)}$ and the diagnosis of hypertension ${ }^{(43)}$ were assessed in subsamples of the cohort.

\section{Assessment of sugar-sweetened beverage consumption}

Participants reported SSB consumption at baseline in the fulllength FFQ and at the 6-year follow-up in a shorter version of this FFQ. SSB consumption was assessed by a previously validated semi-quantitative FFQ in Spain ${ }^{(37-39)}$. In both the baseline assessment and the 6-year follow-up, participants reported the frequency of consumption (one drink $=200 \mathrm{ml}$ ) in the previous year (never, one to three drinks/month, one drink/week, two to four drinks/week, four to six drinks/ week, one drink/d, two to three drinks/d, four to six drinks/ $\mathrm{d}$ and more than six drinks/d). The wording and options of these items were exactly the same at baseline and the 6-year follow-up. We considered the following items as SSB: sugarsweetened carbonated colas and fruit-flavoured carbonated sugar soft drinks assessed in one item of the FFQ. The FFQ also assessed in another item artificially sweetened beverages. Intraclass correlation coefficients between the FFQ and multiple dietary records were 0.67 for SSB and 0.46 for artificially sweetened beverages ${ }^{(38)}$, and in the reproducibility analyses, the correlation coefficients were 0.69 for SSB and 0.64 for artificially sweetened beverages ${ }^{(38)}$. In the 6-year follow-up questionnaire (Q6), we only assessed some, but not all, food items included in the baseline FFQ.

The change in SSB consumption was calculated as the difference between SSB consumption in the 6-year follow-up questionnaire and in the baseline FFQ. This difference in consumption was classified into quintiles of change (quintile 1 for those participants who decreased most of their consumption and quintile 5 for those participants who increased most of their consumption), considering the first quintile as the reference category. We did not include bottled fruit juices as SSB because we did not have the information on consumption at the 6-year follow-up, and according to the last report of the European Fruit Juice Association, almost half (45\%) of the fruit juice sold in Spain was $100 \%$ juice content ${ }^{(44)}$.

\section{Statistical analysis}

The cumulative incidence of the MetS was computed for each quintile of the change in SSB consumption. To avoid the confounding effect of other variables simultaneously associated with the outcome and the main exposure, we used unconditional logistic regression models.
We also assessed the association between the quintiles of the change in SSB consumption and each criterion for the MetS, and the association between the quintiles of the change in SSB consumption and weight change. Linear trend tests were calculated using the median change in SSB consumption of each quintile and by introducing this new variable as a continuous one in the models.

We evaluated the effect modification of some variables such as sex or age through likelihood ratio tests for the product term introduced in fully adjusted models.

As secondary analyses, we assessed the association between baseline SSB and artificially sweetened beverages and the incidence of the MetS.

The statistical software package SPSS for Windows version 14.0 (SPSS, Inc.) was used for statistical analyses. $P$ values were based on the two-tailed test and $P$ values less than 0.05 were considered as statistically significant.

As sensitivity analyses, we estimated the full-adjusted OR for the quintiles of the change in SSB consumption after modifying several assumptions: (1) using the Adult Treatment Panel (ATP)-III criteria for the MetS; (2) using the International Diabetes Federation criteria for the MetS; (3) adopting different limits for allowable total energy intake; (4) excluding participants with diabetes, cancer or CVD at baseline. In the same line, we calculated the hazard ratio for the quintiles of the change in SSB consumption using Cox regression models instead of OR calculated by unconditional logistic regression.

According to the ATP-III criteria for the MetS, the diagnosis of the MetS needs the presence of three of the following five factors: abdominal obesity (more than $102 \mathrm{~cm}$ in men or more than $88 \mathrm{~cm}$ in women); elevated TAG (equal or more than $1500 \mathrm{mg} / \mathrm{l}$ ); reduced HDL-cholesterol (less than $400 \mathrm{mg} / \mathrm{l}$ in men or less than $500 \mathrm{mg} / \mathrm{l}$ in women); elevated blood pressure (equal or more than $130 / 85 \mathrm{mmHg}$ ); elevated fasting glucose (impaired fasting glucose equal or more than $1100 \mathrm{mg} / \mathrm{l}$ or type 2 diabetes mellitus) ${ }^{(45)}$.

According to the International Diabetes Federation criteria for the MetS, the diagnosis of the MetS needs the presence of waist circumference more than $94 \mathrm{~cm}$ in men and more than $80 \mathrm{~cm}$ in women plus any two of other criteria: raised TAG (more than $1500 \mathrm{mg} / \mathrm{l}$ or specific treatment for this lipid abnormality); reduced HDL-cholesterol (less than $40 \mathrm{mg} / \mathrm{d}$ in men or less than $500 \mathrm{mg} / \mathrm{l}$ in women or specific treatment for this lipid abnormality); raised blood pressure (systolic more than $130 \mathrm{mmHg}$ or diastolic more than $85 \mathrm{mmHg}$ or treatment of previously diagnosed hypertension); raised fasting plasma glucose (fasting plasma glucose more than $1000 \mathrm{mg} / 1$ or previously diagnosed type 2 diabetes) ${ }^{(46)}$.

\section{Results}

The baseline characteristics of the patients lost to follow-up did not substantially differ from those who completed the follow-up (data not shown). The characteristics of the participants according to their quintiles of the change in SSB consumption are presented in Table 1 . Those participants who increased their SSB consumption the most were more likely 
to be men, and, on average, they presented a lower adherence to the Mediterranean dietary pattern.

\section{Incidence of the metabolic syndrome}

During a median follow-up of 6 years, we identified 361 incident cases of the MetS among the 8157 young, middle-aged participants (mean age 36 years old) and initially free of the MetS.

Table 2 shows the incidence of the MetS according to the quintiles of the change in SSB consumption. After adjusting for age and sex, participants in the highest quintile of the increase in SSB consumption presented a significantly greater risk of developing the MetS (OR 2.2, $95 \%$ CI 1.4, 3.4; $P$ for trend $=0.003)$ compared with those in the lowest quintile. This association persisted (OR 2.2, $95 \%$ CI 1.4, 3.5; $P$ for trend $=0.003$ ) even after the adjustment for potential dietary and non-dietary confounding factors (baseline BMI, smoking, physical activity, alcohol intake, total energy intake, baseline SSB consumption, red meat, French fries, fast food and adherence to the Mediterranean dietary pattern) and even after the adjustment for the change in weight during the follow-up period (OR 2.0, 95\% CI 1.3, 3.1; $P$ for trend=0.038). When we repeated the analyses using servings (one can $=330 \mathrm{ml}$ ) of change, those participants who increased their consumption of SSB during the follow-up in more than one serving per week increased their risk of developing the MetS by $101 \%$ (adjusted OR 2.01, 95\% CI 1.14, 3.55) in comparison with those participants who decreased their consumption to one serving per week or more.

We did not observe any significant interaction between the changes in SSB consumption and sex $(P$ for interaction $=0.45)$ or age $(P$ for interaction $=0 \cdot 86$ )

In the SUN cohort, the consumption of SSB was very low. Only 405 participants included in the analyses (4.9\%) consumed one or more servings per $\mathrm{d}$ at baseline.
In a secondary analysis, we found no significant association between baseline SSB consumption and incident MetS (data not shown). The consumption of artificially sweetened soft drinks was even lower among the SUN participants. Of these study participants, $75 \%$ never consumed artificially soft drinks, and only $2 \cdot 8 \%$ consumed one or more servings per d. Similarly, we did not find any association between baseline artificially soft drink consumption and the incidence of the MetS.

As a sensitivity analysis, we assessed the association between the changes in SSB consumption between the 6 years of follow-up and baseline (Q6-Q0) and the incidence of the MetS in the 8-year follow-up. We only identified 107 incident cases. We did not observe any significant association.

The direct association between an increase in SSB consumption and the risk of the MetS compared with a decrease in SSB consumption was robust and remained statistically significant in all but one sensitivity analysis (Table 5). The only exception was using the more strict ATP-III criteria of the MetS. Using this definition of the MetS, the changes in SSB intake were not significantly associated with incident MetS. By contrast, when we conducted Cox regression models, the hazard ratio for the fifth quintile of the change in SSB consumption was 1.9 (95\% CI 1.2, 3.0; $P$ for trend 0.032) in comparison with the first quintile of the change in SSB consumption, therefore the association remained statistically significant.

\section{Incidence of individual components of the metabolic syndrome}

Table 3 shows the incidence of the MetS criteria during the follow-up according to the quintiles of the change in SSB consumption. The increases in SSB consumption during the follow-up were associated with a higher risk of developing high blood pressure, central obesity, hypertriacylglycerolaemia

Table 1. Baseline characteristics of the participants according to the quintiles $(Q)$ of the change in sugar-sweetened beverage (SSB) consumption (Mean values and standard deviations*; percentages $\dagger$ )

\begin{tabular}{|c|c|c|c|c|c|c|c|c|c|c|}
\hline & \multicolumn{2}{|c|}{ Q1 } & \multicolumn{2}{|c|}{ Q2 } & \multicolumn{2}{|c|}{ Q3 } & \multicolumn{2}{|c|}{ Q4 } & \multicolumn{2}{|c|}{ Q5 } \\
\hline & Mean & SD & Mean & SD & Mean & SD & Mean & SD & Mean & SD \\
\hline$n$ & \multirow{2}{*}{\multicolumn{2}{|c|}{$\begin{array}{l}1890 \\
<-28.57\end{array}$}} & \multicolumn{2}{|c|}{1334} & \multicolumn{2}{|c|}{1796} & \multirow{2}{*}{\multicolumn{2}{|c|}{$\begin{array}{c}1626 \\
>0 \text { to } 33.81\end{array}$}} & \multirow{2}{*}{\multicolumn{2}{|c|}{$\begin{array}{c}1511 \\
>33.81\end{array}$}} \\
\hline Change in SSB consumption $(\mathrm{ml} / \mathrm{d})$ & & & $-28 \cdot 5$ & to $<0$ & & & & & & \\
\hline Age (years) & $31 \cdot 4$ & 8.4 & 34.9 & $9 \cdot 7$ & $41 \cdot 2$ & 11.5 & $36 \cdot 7$ & $10 \cdot 1$ & 34.5 & $10 \cdot 1$ \\
\hline Women (\%) & \multicolumn{2}{|c|}{$65 \cdot 6$} & \multicolumn{2}{|c|}{71.4} & \multicolumn{2}{|c|}{$74 \cdot 0$} & \multicolumn{2}{|c|}{$64 \cdot 1$} & \multicolumn{2}{|c|}{51.4} \\
\hline Alcohol consumption $(\mathrm{g} / \mathrm{d})$ & $6 \cdot 4$ & $8 \cdot 2$ & 4.9 & $7 \cdot 7$ & $5 \cdot 7$ & 9.5 & $5 \cdot 1$ & 6.9 & $6 \cdot 8$ & 8.9 \\
\hline Baseline SSB consumption $(\mathrm{ml} / \mathrm{d})$ & 109.6 & $119 \cdot 8$ & 26.53 & $35 \cdot 1$ & 0 & NA & 13.5 & 9.9 & 58.6 & $80 \cdot 6$ \\
\hline Baseline BMI $\left(\mathrm{kg} / \mathrm{m}^{2}\right)$ & $22 \cdot 5$ & $2 \cdot 8$ & 22.5 & $2 \cdot 7$ & $22 \cdot 7$ & $2 \cdot 7$ & $22 \cdot 6$ & $2 \cdot 7$ & $23 \cdot 0$ & 2.9 \\
\hline $\begin{array}{l}\text { Leisure-time physical activity } \\
\text { (MET-h/week) }\end{array}$ & $19 \cdot 7$ & $2 \cdot 7$ & $19 \cdot 0$ & $20 \cdot 4$ & $20 \cdot 4$ & $21 \cdot 4$ & $20 \cdot 8$ & 23.4 & $20 \cdot 3$ & $23 \cdot 8$ \\
\hline Total energy intake $(\mathrm{kJ} / \mathrm{d})$ & $10351 \cdot 0$ & $2531 \cdot 3$ & 9913.5 & $2411 \cdot 2$ & 9303.5 & $2540 \cdot 5$ & $9760 \cdot 3$ & $2449 \cdot 7$ & $10316 \cdot 7$ & 2652.7 \\
\hline Mediterranean diet adherence & 3.8 & 1.7 & 4.0 & 1.8 & 4.4 & 1.8 & $4 \cdot 0$ & 1.8 & 1.7 & $1 \cdot 7$ \\
\hline Current smokers (\%) & \multicolumn{2}{|c|}{$30 \cdot 7$} & \multicolumn{2}{|c|}{$23 \cdot 1$} & \multicolumn{2}{|c|}{$18 \cdot 9$} & \multicolumn{2}{|c|}{$20 \cdot 0$} & \multicolumn{2}{|c|}{$27 \cdot 2$} \\
\hline Former smokers (\%) & \multirow{2}{*}{\multicolumn{2}{|c|}{$\begin{array}{l}19 \cdot 6 \\
43.9\end{array}$}} & \multirow{2}{*}{\multicolumn{2}{|c|}{$\begin{array}{l}24 \cdot 4 \\
35 \cdot 1\end{array}$}} & \multirow{2}{*}{\multicolumn{2}{|c|}{$\begin{array}{l}33.8 \\
29.5\end{array}$}} & \multirow{2}{*}{\multicolumn{2}{|c|}{$\begin{array}{l}26 \cdot 1 \\
31 \cdot 0\end{array}$}} & \multirow{2}{*}{\multicolumn{2}{|c|}{$\begin{array}{l}23 \cdot 6 \\
36 \cdot 0\end{array}$}} \\
\hline Snacking between meals (\%) & & & & & & & & & & \\
\hline Television watching (h/week) & $5 \cdot 6$ & $4 \cdot 3$ & $5 \cdot 8$ & $4 \cdot 3$ & 6.0 & 4.4 & 5.9 & 4.4 & 5.9 & 4.4 \\
\hline
\end{tabular}

NA, not applicable; MET, metabolic equivalent

${ }^{\star}$ For continuous variables.

†For categorical variables.

¥ Score proposed by Trichopoulou et al. ${ }^{(40)}(1-9)$ 
Table 2. Incident metabolic syndrome (MetS) according to the quintiles $(\mathrm{Q})$ of the change in sugar-sweetened beverage (SSB) consumption (Median values; numbers and percentages; odds ratios and $95 \%$ confidence intervals)

\begin{tabular}{|c|c|c|c|c|c|c|c|c|c|c|c|}
\hline & \multicolumn{2}{|r|}{ Q1 } & \multicolumn{2}{|c|}{ Q2 } & \multicolumn{2}{|r|}{ Q3 } & \multicolumn{2}{|c|}{ Q4 } & \multicolumn{2}{|c|}{ Q5 } & \multirow[b]{2}{*}{$P$ for trend } \\
\hline & OR & $95 \% \mathrm{Cl}$ & OR & $95 \% \mathrm{Cl}$ & OR & $95 \% \mathrm{Cl}$ & OR & $95 \% \mathrm{Cl}$ & OR & $95 \% \mathrm{Cl}$ & \\
\hline$n$ & \multicolumn{2}{|r|}{1890} & \multicolumn{2}{|c|}{1334} & \multicolumn{2}{|c|}{1796} & \multicolumn{2}{|c|}{1626} & \multicolumn{2}{|c|}{1511} & \\
\hline $\begin{array}{l}\text { Change in SSB } \\
\text { consumption }(\mathrm{ml} / \mathrm{d})\end{array}$ & \multicolumn{2}{|r|}{$\leq-28.57$} & \multicolumn{2}{|c|}{-28.58 to $<0$} & \multicolumn{2}{|c|}{0} & \multicolumn{2}{|c|}{$>0$ to 33.81} & \multicolumn{2}{|c|}{$>33.81$} & \\
\hline \multicolumn{12}{|l|}{ Servings/week* } \\
\hline Median & \multicolumn{2}{|r|}{-1.35} & \multicolumn{2}{|c|}{-0.3} & \multicolumn{2}{|r|}{0} & \multicolumn{2}{|c|}{0.4} & \multicolumn{2}{|c|}{$2 \cdot 4$} & \\
\hline \multicolumn{12}{|l|}{ MetS incidence } \\
\hline$n$ & \multicolumn{2}{|r|}{33} & \multicolumn{2}{|c|}{59} & \multicolumn{2}{|r|}{105} & \multicolumn{2}{|c|}{83} & \multicolumn{2}{|c|}{81} & \\
\hline$\%$ & \multicolumn{2}{|r|}{$1 \cdot 7$} & \multicolumn{2}{|r|}{$4 \cdot 4$} & \multicolumn{2}{|r|}{$5 \cdot 8$} & \multicolumn{2}{|c|}{$5 \cdot 1$} & \multicolumn{2}{|c|}{$5 \cdot 4$} & \\
\hline Crude & 1 & Reference & $2 \cdot 6$ & $1 \cdot 7,4 \cdot 0$ & 3.5 & $2 \cdot 3,5 \cdot 2$ & $3 \cdot 0$ & $2 \cdot 0,4 \cdot 6$ & $3 \cdot 2$ & $2 \cdot 1,4 \cdot 8$ & $<0.001$ \\
\hline Age-sex adjusted & 1 & Reference & $2 \cdot 2$ & $1.4,3.5$ & 1.9 & $1 \cdot 3,3 \cdot 0$ & $2 \cdot 2$ & $1.4,3 \cdot 3$ & $2 \cdot 2$ & $1.4,3.4$ & 0.003 \\
\hline Multivariate $\nmid$ adjusted & 1 & Reference & $2 \cdot 2$ & $1 \cdot 4,3 \cdot 6$ & 1.9 & $1 \cdot 2,3 \cdot 0$ & $2 \cdot 2$ & $1 \cdot 3,3.5$ & $2 \cdot 2$ & $1.4,3.5$ & 0.003 \\
\hline Additionally adjusted & 1 & Reference & $2 \cdot 2$ & $1 \cdot 3,3 \cdot 6$ & 1.8 & $1 \cdot 1,2 \cdot 9$ & 1.9 & $1 \cdot 2,3 \cdot 1$ & $2 \cdot 0$ & $1 \cdot 3,3 \cdot 1$ & 0.038 \\
\hline
\end{tabular}

*One serving: $330 \mathrm{ml}$.

† Adjusted for age, sex, baseline BMI, smoking, physical activity, alcohol intake, soft drink intake at baseline, total energy intake, consumption of red meat, French fries, fast food and adherence to the Mediterranean dietary pattern (score proposed by Trichopoulou et al. ${ }^{(40)}$ ).

$\ddagger$ Also adjusted for weight change between the 6 years of follow-up and baseline.

and impaired fasting glucose. After adjusting for potential confounders, participants in the highest quintile of the increase in SSB consumption presented a significantly higher risk of developing the blood pressure criterion (OR 1.6, 95\% CI 1.3, $2 \cdot 1 ; P$ for trend $<0 \cdot 001)$, the waist circumference criterion
(OR $2 \cdot 3,95 \%$ CI $1 \cdot 9,2 \cdot 7 ; P$ for trend $<0.001$ ), the TAG criterion (OR $1 \cdot 7,95 \%$ CI $1 \cdot 1,2 \cdot 6 ; P$ for trend=0.015) and the fasting glucose criterion (OR 1.6, $95 \%$ CI 1.1, 2.1; $P$ for trend $=0.016)$ in comparison with those who decreased their consumption. An increase in SSB consumption was not

Table 3. Incident metabolic syndrome criteria according to the quintiles $(Q)$ of the change in sugar-sweetened beverage (SSB) consumption (Odds ratios and $95 \%$ confidence intervals)

\begin{tabular}{|c|c|c|c|c|c|c|c|c|c|c|c|}
\hline & \multicolumn{2}{|r|}{ Q1 } & \multicolumn{2}{|c|}{ Q2 } & \multicolumn{2}{|c|}{ Q3 } & \multicolumn{2}{|c|}{ Q4 } & \multicolumn{2}{|c|}{ Q5 } & \multirow[b]{2}{*}{$P$ for trend } \\
\hline & OR & $95 \% \mathrm{Cl}$ & OR & $95 \% \mathrm{Cl}$ & OR & $95 \% \mathrm{Cl}$ & OR & $95 \% \mathrm{Cl}$ & OR & $95 \% \mathrm{Cl}$ & \\
\hline$n$ & \multicolumn{2}{|r|}{1890} & \multicolumn{2}{|c|}{1334} & \multicolumn{2}{|c|}{1796} & \multicolumn{2}{|c|}{1626} & \multicolumn{2}{|c|}{1511} & \\
\hline \multicolumn{12}{|l|}{ Changes (servings/week) ${ }^{\star}$} \\
\hline Median & \multirow{2}{*}{\multicolumn{2}{|c|}{$\begin{aligned} & -1.35 \\
\leq & -28.57\end{aligned}$}} & \multicolumn{2}{|c|}{-0.3} & \multicolumn{2}{|c|}{0} & \multicolumn{2}{|c|}{0.4} & \multicolumn{2}{|c|}{$2 \cdot 4$} & \\
\hline Changes $(\mathrm{ml} / \mathrm{d})$ & & & $-2 \varepsilon$ & 8 to $<0$ & & 0 & & 33.81 & & 3.81 & \\
\hline \multicolumn{12}{|l|}{ Blood pressure criterion } \\
\hline Incident cases $(n)$ & \multicolumn{2}{|r|}{227} & \multicolumn{2}{|c|}{235} & \multicolumn{2}{|c|}{363} & \multicolumn{2}{|c|}{311} & \multicolumn{2}{|c|}{328} & \\
\hline Crude & 1 & Reference & $1 \cdot 6$ & $1.3,1.9$ & 1.9 & $1 \cdot 5,2 \cdot 2$ & $1 \cdot 7$ & $1 \cdot 4,2 \cdot 1$ & 2 & $1 \cdot 7,2 \cdot 4$ & $<0.001$ \\
\hline Age-sex adjusted & 1 & Reference & $1 \cdot 3$ & $1 \cdot 1,1 \cdot 7$ & $1 \cdot 2$ & $0.9,1.4$ & $1 \cdot 3$ & $1.1,1.5$ & 1.6 & $1 \cdot 3,1 \cdot 9$ & $<0.001$ \\
\hline Multivariate $\dagger$ adjusted & 1 & Reference & 1.4 & $1 \cdot 1,1 \cdot 8$ & $1 \cdot 3$ & $1 \cdot 0,1 \cdot 8$ & 1.6 & $1 \cdot 2,2 \cdot 0$ & 1.6 & $1 \cdot 3,2 \cdot 1$ & $<0.001$ \\
\hline \multicolumn{12}{|l|}{ Waist criterion } \\
\hline Incident cases $(n)$ & \multicolumn{2}{|r|}{688} & \multicolumn{2}{|c|}{550} & \multicolumn{2}{|c|}{749} & \multicolumn{2}{|c|}{771} & & 70 & \\
\hline Crude & 1 & Reference & $1 \cdot 2$ & $1 \cdot 1,1.4$ & $1 \cdot 2$ & $1 \cdot 1,1.4$ & 1.6 & $1 \cdot 4,1 \cdot 8$ & 1.8 & $1 \cdot 6,2 \cdot 1$ & $<0.001$ \\
\hline Age-sex adjusted & 1 & Reference & $1 \cdot 1$ & $0.9,1 \cdot 2$ & $0 \cdot 8$ & $0 \cdot 7,1 \cdot 0$ & $1 \cdot 3$ & $1 \cdot 1,1.5$ & $1 \cdot 6$ & $1.4,1.9$ & $<0.001$ \\
\hline Multivariate $†$ adjusted & 1 & Reference & $1 \cdot 3$ & $1 \cdot 1,1 \cdot 6$ & $1 \cdot 2$ & $1 \cdot 0,1 \cdot 4$ & $2 \cdot 1$ & $1 \cdot 8,2 \cdot 6$ & $2 \cdot 3$ & $1 \cdot 9,2 \cdot 7$ & $<0.001$ \\
\hline HDL criterion & & & & & & & & & & & \\
\hline Incident cases $(n)$ & & 86 & & 88 & & 39 & & 97 & & 74 & \\
\hline Crude & 1 & Reference & $1 \cdot 1$ & $0.8,1.6$ & $1 \cdot 1$ & $0.8,1.5$ & $1 \cdot 3$ & $1 \cdot 0,1 \cdot 8$ & $1 \cdot 1$ & $0.8,1.5$ & 0.6 \\
\hline Age-sex adjusted & 1 & Reference & 1.0 & $0.7,1.5$ & 0.9 & $0 \cdot 7,1 \cdot 3$ & $1 \cdot 2$ & $0 \cdot 9,1 \cdot 7$ & $1 \cdot 1$ & $0.8,1.5$ & 0.6 \\
\hline Multivariate $\dagger$ adjusted & 1 & Reference & 0.9 & $0.6,1.4$ & 0.9 & $0.6,1.5$ & $1 \cdot 3$ & $0 \cdot 9,2 \cdot 0$ & 1.0 & $0 \cdot 7,1 \cdot 6$ & 0.7 \\
\hline TAG criterion & & & & & & & & & & & \\
\hline Incident cases $(n)$ & & 68 & & 5 & & 09 & & 91 & & 98 & \\
\hline Crude & 1 & Reference & 1.6 & $1 \cdot 1,2 \cdot 2$ & 1.7 & $1 \cdot 3,2 \cdot 4$ & 1.6 & $1 \cdot 1,2 \cdot 2$ & 1.9 & $1 \cdot 3,2 \cdot 5$ & 0.001 \\
\hline Age-sex adjusted & 1 & Reference & 1.4 & $1 \cdot 0,2 \cdot 0$ & $1 \cdot 2$ & $0.9,1.7$ & $1 \cdot 3$ & $0 \cdot 9,1 \cdot 8$ & 1.5 & $1 \cdot 1,2 \cdot 1$ & 0.018 \\
\hline Multivariate $\dagger$ adjusted & 1 & Reference & $1 \cdot 2$ & $0 \cdot 7,2 \cdot 1$ & $1 \cdot 2$ & $0 \cdot 7,2 \cdot 0$ & 1.4 & $0 \cdot 9,2 \cdot 3$ & 1.7 & $1 \cdot 1,2 \cdot 6$ & 0.015 \\
\hline Fasting glucose criterion & & & & & & & & & & & \\
\hline Incident cases $(n)$ & & 105 & & 01 & & 98 & & 61 & & 46 & \\
\hline Crude & 1 & Reference & 1.4 & $1 \cdot 1,1 \cdot 8$ & $2 \cdot 1$ & $1 \cdot 6,2 \cdot 7$ & 1.9 & $1.4,2.4$ & 1.8 & $1.4,2.4$ & $<0.001$ \\
\hline Age-sex adjusted & 1 & Reference & $1 \cdot 2$ & $0.9,1.6$ & $1 \cdot 3$ & $1 \cdot 0,1 \cdot 7$ & 1.4 & $1 \cdot 1,1 \cdot 8$ & 1.4 & $1 \cdot 1,1 \cdot 8$ & 0.022 \\
\hline Multivariate $\dagger$ adjusted & 1 & Reference & $1 \cdot 3$ & $0.9,1.9$ & $1 \cdot 3$ & $0.9,1.9$ & 1.6 & $1 \cdot 1,2 \cdot 3$ & 1.6 & $1 \cdot 1,2 \cdot 2$ & 0.016 \\
\hline
\end{tabular}

*One serving: $330 \mathrm{ml}$.

†Adjusted for age, sex, baseline BMI, smoking, physical activity, alcohol intake, soft drink intake at baseline, total energy intake, consumption of red meat, French fries, fast food consumption and adherence to the Mediterranean dietary pattern (score proposed by Trichopoulou et al. ${ }^{(40)}$ ). 
associated with a higher risk of developing the HDL criterion (OR $1 \cdot 0,95 \%$ CI $0 \cdot 7,1 \cdot 6 ; P$ for trend $=0 \cdot 7$ ).

In addition, the changes in SSB consumption were positively associated with weight changes. Participants in the group with the highest increase in SSB consumption presented an average $1.3(95 \%$ CI $1.1,1.6) \mathrm{kg}$ greater weight gain than the group with the highest decrease in SSB consumption after the 6 years of follow-up (Table 4).

As secondary analyses, we assessed the association between baseline quintiles of SSB consumption and the incidence of each criterion of the MetS. We found that a higher baseline consumption of SSB was significantly associated with a higher risk of developing the waist circumference criterion during the follow-up (adjusted OR 1·18, 95\% CI 1.01, 1.37). We did not find any other significant association between SSB consumption and the rest of the criteria.

\section{Discussion}

In the present prospective study of young, middle-aged, freeliving university graduates from a Mediterranean country, an increase in SSB consumption during the follow-up (when compared with reduced consumption) was associated not only with a higher risk of developing the MetS but also with the risk of developing four of the five defining criteria for the MetS: high blood pressure; central obesity; hypertriacylglycerolaemia; and impaired fasting glucose.

Among the 8157 participants included in the present study, we observed 361 incident cases of the MetS. This incidence proportion of the MetS is lower than those described in the general population of Spain ${ }^{(8,9)}$ as it is to be expected in a cohort of young, middle-aged, active adults, with a low baseline BMI and a high educational level. Some studies have shown that a low social class and a low educational level are associated with a higher prevalence of the $\operatorname{MetS}^{(8,10)}$.

The present study is partially consistent with previous results found in three prospective cohorts in the context of a North American population ${ }^{(26,29,30)}$. All of these three previous longitudinal studies, whose results have been summarised in a published meta-analysis ${ }^{(31)}$, found that SSB consumption is a risk factor for developing the MetS. However, we did not find a direct association between baseline SSB consumption and a higher risk of developing the MetS. By contrast, we found an association between the changes in consumption during the follow-up and the risk of developing the MetS $(P=0 \cdot 006)$. There are some differences between those studies and our cohort that may explain these differences in the results. First, the subjects included in these cohorts are different: in the SUN Project, we recruited only Spanish university graduates; in the Framingham Offspring Study, the participants were white Americans ${ }^{(26)}$; in the Multi-Ethnic Study of Atherosclerosis study, the participants were Caucasian, African American, Hispanic and Chinese ${ }^{(29)}$; in the Atherosclerosis Risk in Communities (ARIC) study, the cohort was formed by white and black American men and women ${ }^{(30)}$. Second, our cohort is younger than the other cohorts. Third, in the SUN cohort, the incidence of the MetS was only $4.4 \%$ during the 6 years of follow-up, but it was $18.7 \%$ after a 4-year follow-up in the Framingham Offspring Study, and $12 \cdot 8 \%$ in the Multi-Ethnic Study of Atherosclerosis study after a 5-year follow-up and 39.8\% after a 9-year follow-up in the ARIC study. Finally, the distribution of baseline SSB consumption was very different too, and while in the SUN cohort, only $1 \cdot 1 \%$ of participants consumed two or more servings of SSB per $d$, in the Framingham Offspring Study, this percentage was $13.8 \%$, in the Multi-Ethnic Study of Atherosclerosis study $13.6 \%$ of participants and in the ARIC study $33 \%$ of participants consumed a median of one serving per $\mathrm{d}$.

Heidemann et al. ${ }^{(47)}$ found that a dietary pattern high in refined grains, processed meat, red meat, high-sugar beverages, eggs, potatoes, beer, sweets and cakes, snacks and butter was associated with a higher prevalence of abdominal obesity, hypertension, hypertriacylglycerolaemia and the MetS in a cross-sectional study of a nationally representative sample of 4025 German adults. Interestingly, the present results are in accordance with recent results from the Coronary Artery Risk Development in Young Adults cohort ${ }^{(48)}$ reporting that baseline SSB consumption was associated with several components of the MetS but not with the incidence of the MetS as a whole.

Table 4. Estimates for subsequent weight change $(\mathrm{kg})$ according to the quintiles $(\mathrm{Q})$ of the change in sugar-sweetened beverage (SSB) consumption ( $\beta$ Regression coefficients and 95\% confidence intervals; mean values and standard deviations)

\begin{tabular}{|c|c|c|c|c|c|c|c|c|c|c|c|}
\hline & \multicolumn{2}{|r|}{ Q1 } & \multicolumn{2}{|r|}{ Q2 } & \multicolumn{2}{|r|}{ Q3 } & \multicolumn{2}{|r|}{ Q4 } & \multicolumn{2}{|c|}{ Q5 } & \multirow[b]{2}{*}{$P$ for trend } \\
\hline & $\beta$ & $95 \% \mathrm{Cl}$ & $\beta$ & $95 \% \mathrm{Cl}$ & $\beta$ & $95 \% \mathrm{Cl}$ & $\beta$ & $95 \% \mathrm{Cl}$ & $\beta$ & $95 \% \mathrm{Cl}$ & \\
\hline$n$ & & 1890 & & 1334 & & 1796 & & 1626 & & 511 & \\
\hline Changes in SSB consumption $(\mathrm{ml} / \mathrm{d})$ & & $\leq-28.57$ & $-2 \varepsilon$ & 58 to $<0$ & & 0 & & to 33.81 & & 33.81 & \\
\hline Median* & & $-1 \cdot 35$ & & -0.3 & & 0 & & 0.4 & & $2 \cdot 4$ & \\
\hline \multicolumn{12}{|l|}{ Differences in weight change $(\mathrm{kg})$} \\
\hline Mean & & 1.21 & & 1.47 & & 1.16 & & 1.91 & & .36 & \\
\hline SD & & 3.94 & & 3.94 & & 3.87 & & 4.52 & & .88 & \\
\hline Crude & 0 & Reference & $2 \cdot 6$ & $1 \cdot 7,4 \cdot 0$ & 3.5 & $2 \cdot 3,5 \cdot 2$ & $3 \cdot 0$ & $2 \cdot 0,4 \cdot 6$ & $3 \cdot 2$ & $2 \cdot 1,4 \cdot 8$ & $<0.001$ \\
\hline Age-sex adjusted & 0 & Reference & 0.4 & $0.1,0.7$ & 0.3 & $0.0,0.6$ & 0.9 & $0.6,1.2$ & 1.3 & $1.0,1.6$ & $<0.001$ \\
\hline Multivariate† adjusted & 0 & Reference & 0.5 & $0.2,0.9$ & 0.5 & $0.2,0.8$ & $1 \cdot 1$ & $0.8,1.4$ & $1 \cdot 3$ & $1 \cdot 1,1 \cdot 6$ & $<0.001$ \\
\hline
\end{tabular}

*One serving: $330 \mathrm{ml}$.

† Adjusted for age, sex, baseline BMI, smoking, physical activity, alcohol intake, soft drink intake at baseline, total energy intake, consumption of red meat, French fries, fast food consumption and adherence to the Mediterranean dietary pattern (score proposed by Trichopoulou et al. ${ }^{(40)}$ ). 
The consumption of SSB was very low among our participants. In spite of this, we found that an increase in the consumption of SSB was a risk factor for the MetS in a cohort of young adults. A relatively small increase in SSB consumption (more than one serving: $330 \mathrm{ml} /$ week) was associated with a significantly higher risk of developing the MetS and its conditions in comparison with participants who decreased their consumption to more than one serving per week. In fact, changes from repeated measurements of dietary habits controlling for baseline exposure are useful in assessing the effects of the changes in dietary intakes over time on the development of chronic diseases such as the MetS in a cohort study ${ }^{(49)}$. This is a methodological strength of our assessment.

There are different mechanisms that can explain the higher risk of the MetS associated with greater SSB consumption. A larger consumption of added sweeteners such as high-fructose corn syrup (the primary sweetener in SSB) can lead to weight gain, increased insulin resistance ${ }^{(50,51)}$, lower HDL-cholesterol $^{(27,52)}$ and increased TAG levels ${ }^{(27,53)}$. Consumption of liquids is associated with a lesser degree of dietary compensation (the adjustment in energy intake made in subsequent meals in response to food intake) ${ }^{(54)}$ because beverages hold weak satiety properties in comparison with solid foods. Some studies have suggested that this property might be due to the food form (i.e. liquid in beverage form compared with a semisolid or solid physical state) and not to the macronutrient or energy source (i.e. carbohydrate, fat or protein). In any case, both mechanisms are likely to be synergistic and responsible for the association between energy-yielding beverage consumption and positive energy balance ${ }^{(55)}$.

Individuals with a greater intake of SSB have also a dietary pattern characterised by a greater intake of energy and saturated and trans-fats, a lower consumption of fibre ${ }^{(56)}$ and dairy products $^{(57)}$, and a more sedentary lifestyle ${ }^{(58)}$. Nevertheless, in the present study, we adjusted for total energy intake, smoking, physical activity, alcohol intake, French fries, red meat, fast food and adherence to a Mediterranean dietary pattern in a multivariable analysis, and still we observed a significant association between the changes in SSB consumption and the risk of developing the MetS and its component criteria.

SSB consumption has been associated with obesity ${ }^{(59)}$. Waist circumference is a criterion of the MetS; moreover, some MetS definitions consider central obesity as the more important MetS criterion ${ }^{(46)}$. However, when we adjusted for weight change during the follow-up, we observed that the association between SSB and the MetS still remained significant, although the magnitude of the association decreased. Therefore, other potential causal pathways may contribute to explaining the relationship between the increases in SSB consumption and the MetS in addition to weight gain.

It is conceivable, though, that because of the observational nature of the present study, we cannot completely rule out residual confounding caused by lifestyle factors not adjusted for in the present analyses.

Our participants are not representative of the general Spanish population. We restricted our cohort to highly educated participants to obtain a better quality of self-reported information, to improve the retention rate and to minimise 
confounding by educational level, and therefore by socioeconomic status $^{(60)}$. We estimated the relative effects, comparing MetS incidence between the different categories of the change in SSB consumption within the same homogeneous educational-level population.

Inherent to nutritional epidemiology methods, we have to point out the possibility of some degree of misclassification in the dietary assessment. However, we used a FFQ previously validated in Spain ${ }^{(38,39)}$. In addition, we would expect this misclassification to be non-differential, and therefore it may drive the association towards the null value.

Other possible causes of concern could be related to the differential losses of the follow-up; however, the baseline characteristics of the participants lost to follow-up did not substantially differ from those who completed the follow-up (data not shown).

The present study has important strengths such as the repeated measurement of the dietary exposure of interest, the relatively large number of participants, the long followup period, the previously published validation studies assessing the validity of our methods, the control for an important number of potential confounders (including the overall dietary pattern) and the good quality of the self-reported data of our highly educated volunteers.

In summary, even a relatively small increase in SSB consumption was associated with a higher risk of developing the MetS and other metabolic disorders in a cohort of young, middle-aged Spanish university graduates. However, since the incidence of the MetS was low, further studies should be conducted to confirm these findings.

\section{Acknowledgements}

The authors thank the collaboration of the participants in the SUN Project and the assistance of Dr Joan Fernandez-Ballart for his information on specific validity data of SSB in the last validation study of the FFQ. The SUN Project has received funding from the Spanish Government (Grants PI01/0619, PI030678, PI040233, PI042241, PI050976, PI070240, PI070312, PI081943, PI080819, PI1002658, PI1002293, PND2010/87, RD06/0045 and G03/140), the Navarra Regional Government (36/2001, $43 / 2002,41 / 2005,36 / 2008$ and 45/2011) and the University of Navarra. M. T. B.-L. participated in the study design, statistical analyses, data interpretation and manuscript drafting. M. A. M.-G. participated in the data interpretation, funding, concept and design of the study. A. F.-M., J. J. B. and I. Z. participated in the study design and data interpretation. M. B.-R. participated in the study design, statistical analyses, data interpretation, funding and manuscript drafting. All authors revised the manuscript for important intellectual content, and read and approved the final version of the manuscript. The authors declare that they have no competing interests.

\section{References}

1. Ford ES, Giles WH \& Mokdad AH (2004) Increasing prevalence of the metabolic syndrome among US adults. Diabetes Care 27, 2444-2449.
2. Athyros VG, Ganotakis ES, Elisaf M, et al. (2005) The prevalence of the metabolic syndrome using the National Cholesterol Educational Program and International Diabetes Federation definitions. Curr Med Res Opin 21, 1157-1159.

3. Ford ES, Giles WH \& Dietz WH (2002) Prevalence of the metabolic syndrome among US adults: findings from the Third National Health and Nutrition Examination Survey. JAMA 287, 356-359.

4. Ford ES, Li C \& Zhao G (2010) Prevalence and correlates of metabolic syndrome based on a harmonious definition among adults in the US. J Diabetes 2, 180-193.

5. Alkerwi A, Donneau AF, Sauvageot N, et al. (2011) Prevalence of the metabolic syndrome in Luxembourg according to the Joint Interim Statement definition estimated from the ORISCAV-LUX study. BMC Public Health 11, 4.

6. Lim S, Shin H, Song JH, et al. (2011) Increasing prevalence of metabolic syndrome in Korea: the Korean National Health and Nutrition Examination Survey for 1998-2007. Diabetes Care 34, 1323-1328.

7. Delavari A, Forouzanfar MH, Alikhani S, et al. (2009) First nationwide study of the prevalence of the metabolic syndrome and optimal cutoff points of waist circumference in the Middle East: the national survey of risk factors for noncommunicable diseases of Iran. Diabetes Care 32, 1092-1097.

8. Alegria E, Cordero A, Laclaustra M, et al. (2005) Prevalence of metabolic syndrome in the Spanish working population: MESYAS registry. Rev Esp Cardiol 58, 797-806.

9. Fernández-Bergés D, Cabrera de León A, Sanz $\mathrm{H}$, et al. (2012) Metabolic syndrome in Spain: prevalence and coronary risk associated with harmonized definition and WHO proposal. DARIOS study. Rev Esp Cardiol 65, 241-248.

10. Brouwer BG, Visseren FL, van der Graaf Y, et al. (2007) The effect of leisure-time physical activity on the presence of metabolic syndrome in patients with manifest arterial disease. The SMART study. Am Heart J 154, 1146-1152.

11. Lustig RH, Schmidt LA \& Brindis CD (2012) Public health: the toxic truth about sugar. Nature 482, 27-29.

12. Popkin BM (2010) Patterns of beverage use across the lifecycle. Physiol Behav 100, 4-9.

13. Barquera S, Hernandez-Barrera L, Tolentino ML, et al. (2008) Energy intake from beverages is increasing among Mexican adolescents and adults. $J$ Nutr 138, 2454-2461.

14. Welsh JA, Sharma AJ, Grellinger L, et al. (2011) Consumption of added sugars is decreasing in the United States. Am J Clin Nutr 94, 726-734.

15. Malik VS, Popkin BM, Bray GA, et al. (2010) Sugar-sweetened beverages, obesity, type 2 diabetes mellitus, and cardiovascular disease risk. Circulation 121, 1356-1364.

16. Ludwig DS, Peterson KE \& Gortmaker SL (2010) Relation between consumption of sugar-sweetened drinks and childhood obesity: a prospective, observational analysis. Lancet 357, 505-508

17. Schulze MB, Manson JE, Ludwig DS, et al. (2004) Sugarsweetened beverages, weight gain, and incidence of type 2 diabetes in young and middle-aged women. JAMA 292, 927-934.

18. Malik VS, Popkin BM, Bray GA, et al. (2010) Sugar-sweetened beverages and risk of metabolic syndrome and type 2 diabetes: a meta-analysis. Diabetes Care 33, 2477-2483.

19. Winkelmayer WC, Stampfer MJ, Willett WC, et al. (2005) Habitual caffeine intake and the risk of hypertension in women. JAMA 294, 2330-2335.

20. de Koning L, Malik VS, Kellogg MD, et al. (2012) Sweetened beverage consumption, incident coronary heart disease and biomarkers of risk in men. Circulation 125, 1735-1741. 
21. Parks EJ \& Hellerstein MK (2000) Carbohydrate-induced hypertriacylglycerolemia: historical perspective and review of biological mechanisms. Am J Clin Nutr 71, 412-433.

22. Smith JB, Niven BE \& Mann JI (1996) The effect of reduced extrinsic sucrose intake on plasma triglyceride levels. Eur J Clin Nutr 50, 498-504.

23. Surwit RS, Feinglos MN, McCaskill CC, et al. (1997) Metabolic and behavioral effects of a high-sucrose diet during weight loss. Am J Clin Nutr 65, 908-915.

24. Swanson JE, Laine DC, Thomas W, et al. (1992) Metabolic effects of dietary fructose in healthy subjects. Am J Clin Nutr 55, 851-856.

25. Jurgens H, Haass W, Castaneda TR, et al. (2005) Consuming fructose-sweetened beverages increases body adiposity in mice. Obes Res 13, 1146-1156.

26. Dhingra R, Sullivan L, Jacques PF, et al. (2007) Soft drink consumption and risk of developing cardiometabolic risk factors and the metabolic syndrome in middle-aged adults in the community. Circulation 116, 480-488.

27. Høstmark AT \& Tomten SE (2009) Cola intake and serum lipids in the Oslo Health Study. Appl Physiol Nutr Metab 34, 901-906.

28. Høstmark AT (2010) The Oslo Health Study: a Dietary Index estimating high intake of soft drinks and low intake of fruits and vegetables was positively associated with components of the metabolic syndrome. Appl Physiol Nutr Metab 35 , $816-825$.

29. Nettleton JA, Lutsey PL, Wang Y, et al. (2009) Diet soda intake and risk of incident metabolic syndrome and type 2 diabetes in the Multi-Ethnic Study of Atherosclerosis (MESA). Diabetes Care 32, 688-694.

30. Lutsey PL, Steffen LM \& Stevens J (2008) Dietary intake and the development of the metabolic syndrome: the Atherosclerosis Risk in Communities study. Circulation 117, 754-761.

31. Malik VS, Popkin BM, Bray GA, et al. (2010) Sugar-sweetened beverages and risk of metabolic syndrome and type 2 diabetes. Diabetes Care 33, 2477-2483.

32. Martinez-Gonzalez MA, Sanchez-Villegas A, De Irala J, et al. (2002) Mediterranean diet and stroke: objectives and design of the SUN project. Seguimiento Universidad de Navarra. Nutr Neurosci 5, 65.

33. Segui-Gomez M, de la Fuente C, Vazquez Z, et al. (2006) Cohort profile: the 'Seguimiento Universidad de Navarra' (SUN) study. Int J Epidemiol 35, 1417-1422.

34. Alberti KG, Eckel RH, Grundy SM, et al. (2009) Harmonizing the metabolic syndrome: a joint interim statement of the International Diabetes Federation Task Force on Epidemiology and Prevention; National Heart, Lung, and Blood Institute; American Heart Association; World Heart Federation; International Atherosclerosis Society; and International Association for the Study of Obesity. Circulation 120, $1640-1645$.

35. Fernandez-Montero A, Beunza JJ, Bes-Rastrollo $\mathrm{M}$, et al. (2011) Validación de los componentes del síndrome metabólico autodeclarados en un estudio de cohortes. Spanish (Validation of the self-reported metabolic syndrome components in a cohort study). Gac Sanit 25, 303-307.

36. Barrio-Lopez MT, Bes-Rastrollo M, Beunza JJ, et al. (2011) Validation of metabolic syndrome using medical records in the SUN cohort. BMC Public Health 11, 867.

37. Martín-Moreno JM, Boyle P, Gorgojo L, et al. (1993) Development and validation of a food frequency questionnaire in Spain. Int J Epidemiol 22, 512-519.

38. Fernandez-Ballart JD, Piñol JL, Zazpe I, et al. (2010) Relative validity of a semi-quantitative food-frequency questionnaire in an elderly Mediterranean population of Spain. Br J Nutr 103, 1808-1816.

39. de la Fuente-Arrillaga C, Ruiz ZV, Bes-Rastrollo M, et al. (2010) Reproducibility of an FFQ validated in Spain. Public Health Nutr 13, 1364-1372.

40. Trichopoulou A, Costacou T, Bamia C, et al. (2003) Adherence to a Mediterranean diet and survival in a Greek population. N Engl J Med 348, 2599-2608.

41. Bes-Rastrollo M, Perez Valdivieso JR, Sanchez-Villegas A, et al. (2005) Validación del peso e índice de masa corporal auto-declarados de los participantes de una cohorte de graduados universitarios. Spanish (Validation of the selfreported weight and body mass index of participants in a cohort of university graduates). Rev Esp Obes 3, 352-358.

42. Martinez-Gonzalez MA, Lopez-Fontana C, Varo JJ, et al. (2005) Validation of the Spanish version of the physical activity questionnaire used in the Nurses' Health Study and Health Professionals' Follow-up study. Public Health Nutr 8, 920-927.

43. Alonso A, Beunza JJ, Delgado-Rodriguez M, et al. (2005) Validation of self reported diagnosis of hypertension in a cohort of university graduates in Spain. BMC Public Health 5, 94 .

44. European Fruit Juice Association (2012) Liquid Fruit. Market Report. Brussels: AIJN. http://www.aijn.org/pages/main/factsfigures.html (accessed February 2013).

45. Grundy SM, Cleeman JI, Daniels SR, et al. (2005) American Heart Association; National Heart, Lung, and Blood Institute. Diagnosis and management of the metabolic syndrome: an American Heart Association/National Heart, Lung, and Blood Institute Scientific Statement. Circulation 112, $2735-2752$.

46. Alberti KG, Zimmet P \& Shaw J (2005) IDF Epidemiology Task Force Consensus Group. The metabolic syndrome: a new worldwide definition. Lancet 366, 1059-1062.

47. Heidemann C, Scheidt-Nave C, Richter A, et al. (2011) Dietary patterns are associated with cardiometabolic risk factors in a representative study population of German adults. $\mathrm{Br}$ J Nutr 106, 1253-1262.

48. Duffey KJ, Gordon-Larsen P, Steffen LM, et al. (2010) Drinking caloric beverages increases the risk of adverse cardiometabolic outcomes in the Coronary Artery Risk Development in Young Adults (CARDIA) Study. Am J Clin Nutr 92, 954-959.

49. Hu FB (2008) Analytic epidemiologic designs in obesity research. In Obesity Epidemiology, 1st ed., pp. 26-37 [FB $\mathrm{Hu}$, editor]. New York, NY: Oxford University Press.

50. Bray GA, Nielsen SJ \& Popkin BM (2004) Consumption of high-fructose corn syrup in beverages may play a role in the epidemic of obesity. Am J Clin Nutr 79, 537-543.

51. Elliott SS, Keim NL, Stern JS, et al. (2002) Fructose, weight gain, and the insulin resistance syndrome. Am J Clin Nutr 76, 911-922.

52. Frost G, Leeds AA, Dore CJ, et al. (1999) Glycaemic index as a determinant of serum HDL-cholesterol concentration. Lancet 353, 1045-1048.

53. Willett W, Manson J \& Liu S (2002) Glycemic index, glycemic load, and risk of type 2 diabetes. Am J Clin Nutr 76, $274-280$.

54. Cassady BA, Considine RV \& Mattes RD (2012) Beverage consumption, appetite, and energy intake: what did you expect? Am J Clin Nutr 95, 587-593.

55. Mattes RD (2006) Beverages and positive energy balance: the menace is the medium. Int J Obes 30, 60-65.

56. Pereira MA, Kartashov AI, Ebbeling CB, et al. (2005) Fastfood habits, weight gain, and insulin resistance (the 
CARDIA study): 15-year prospective analysis. Lancet 365 , 36-42.

57. Rampersaud GC, Bailey LB \& Kauwell GP (2003) National survey beverage consumption data for children and adolescents indicate the need to encourage a shift toward more nutritive beverages. J Am Diet Assoc 103, 97-100.

58. Hu FB, Li TY, Colditz GA, et al. (2003) Television watching and other sedentary behaviors in relation to risk of obesity and type 2 diabetes mellitus in women. JAMA $\mathbf{2 8 9}$, 1785-1791.

59. Qi Q, Chu AY, KangJH, et al. (2012) Sugar-sweetened beverages and genetic risk of obesity. N Engl J Med 367, 1387-1396.

60. Rothman KJ, Greenland S \& Lash TL (2008) Design strategies to improve study accuracy. In Modern Epidemiology, 3rd ed., pp. 168-182 [KJ Rothman, S Greenland and TL Lash, editors]. Philadelphia: Lippincott Williams and Wilkins. 